\title{
ELABORACIÓN DE HORMIGONES QUE MINIMICEN EL IMPACTO AL AMBIENTE, EMPLEANDO NEUMÁTICOS USADOS DE VEHÍCULOS LIVIANOS Y PESADOS, EN LA PROVINCIA DE SANTA ELENA
}

\author{
Armando Saltos ${ }^{1}$, Humberto Guerrero ${ }^{2}$ \\ Linda Ordóñez ${ }^{3}$, Miguel Suárez ${ }^{3}$ \\ ${ }^{1}$ Centro de Investigación de Geociencias (CIGEO) \\ ${ }^{2}$ Facultad de Ciencias de la Ingeniería \\ ${ }^{3}$ Universidad Estatal Península de Santa Elena (UPSE) \\ Campus La Libertad, vía principal Santa Elena - La Libertad \\ La Libertad-Ecuador \\ armandosaltos@hotmail.com
}

\begin{abstract}
Resumen
Las llantas de desecho son consideradas un foco de riesgo ambiental y sanitario a nivel mundial, por ejemplo en Ecuador se desecha al año, según la Corporación para la Promoción Proactiva de Inversiones (INVEC), cerca de 2,4 millones de neumáticos de diversos tipos, lo que equivale a 55.000 toneladas. Un menor porcentaje de ellos son reutilizados para el reencauchado, pero la gran mayoría es incinerada o depositada en basureros a cielo abierto, lo que supone una amenaza contra el medio ambiente y riesgos a la salud. El proyecto busca la creación de un diseño de hormigón con partículas provenientes de neumáticos usados, que después de un proceso de selección de los neumáticos a usar, de una trituración controlada y una dosificación acorde con los parámetros de las normas internacionales de diseño de hormigón del Instituto Americano del Concreto (ACI) y de la Norma Ecuatoriana de la Construcción (NEC), se obtenga un hormigón de resistencias aceptables que puedan ser empleadas en: calles de bajo tránsito, banquetas y zonas peatonales y otras estructuras de hormigón, minimizando así el impacto ambiental que se produce por el mal manejo de llantas usadas en la provincia de Santa Elena.
\end{abstract}

Palabras Claves: Reciclaje, ambiente, diseño, hormigón, normas.

\begin{abstract}
Waste from rubbers tires are considered to be an environmental and sanitary risk worldwide. As an example in Ecuador, every year nearly 2.4 million rubber tires of various types are discarded as trash, which is equivalent to 55000 tons, according to the Proactive Corporation for Investment Promotion (INVEC). A small porcentage of the waste, are reused to be re-rubberized. But the majority is incinerate or deposited in dumping grounds open to the environment, which is a huge risk to the environment and the health of individuals. The project seeks to create a design of concrete including particles of rubber from discarded tires. The tires will undergo a rigorous selection process, a controlled threading process, and a fabrication according to the specifications of the international standards of concrete of the American Concrete Institute (ACI) and the Construction Ecuatorian Standards (NEC), and based on these specifications, the goal is to obtain an acceptable concrete apt to be used in low traffic streets, sidewalks, pedestrian zones, and other concrete structures, minimizing the environmental impact to the misuse of tires in the province of Santa Elena.
\end{abstract}

Keywords: Recycling, environment, design, concrete, norms. 


\section{Introducción}

Las llantas de desecho son consideradas un foco de riesgo ambiental y sanitario a nivel mundial, por el riesgo de un incendio o por quedar simple y sencillamente apiladas por años ya que se estiman que tardan 500 años en degradarse, generando riesgo a que se incrementen las emisiones a la atmósfera de diversas sustancias peligrosas.

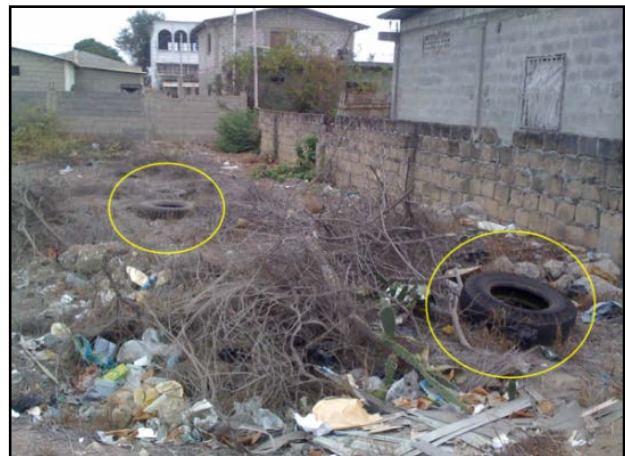

Foto 1.1.- Llantas en terreno baldío en vía Santa Elena-La Libertad.

Fuente Foto: Investigadores CIGEO-Julio/2014

Ecuador desecha al año, según la Corporacion para la Promoción Proactiva de Inversiones (INVEC), cerca de 2,4 millones de neumáticos de diversos tipos, lo que equivale a 55.000 toneladas. Un menor porcentaje de ellos son reutilizados para el reencauchado, principalmente en vehículos de carga pesada, pero la gran mayoría es incinerada o depositada en basureros a cielo abierto, lo que supone una amenaza contra el medio ambiente y riesgos a la salud.

La ineficacia en la creación y aplicación de ordenanzas que regulen la disposición final de las llantas usadas, siendo uno de los desperdicios más difíciles de manejar, que al terminar su ciclo de vida útil son eliminadas; en el Ecuador "sólo 30\% de los municipios tienen rellenos sanitarios" (INVEC), de los cuales no muchos tienen un lugar adecuado para la disposición de dichos desperdicios.

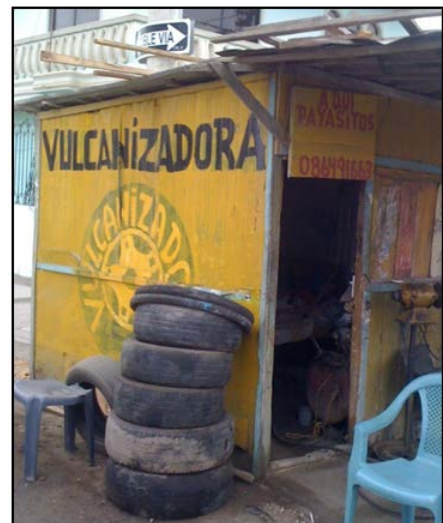

Foto 1.2.- Deposición de llantas en vulcanizadora. Santa Elena.

Fuente: Investigadores CIGEO-Julio/2014

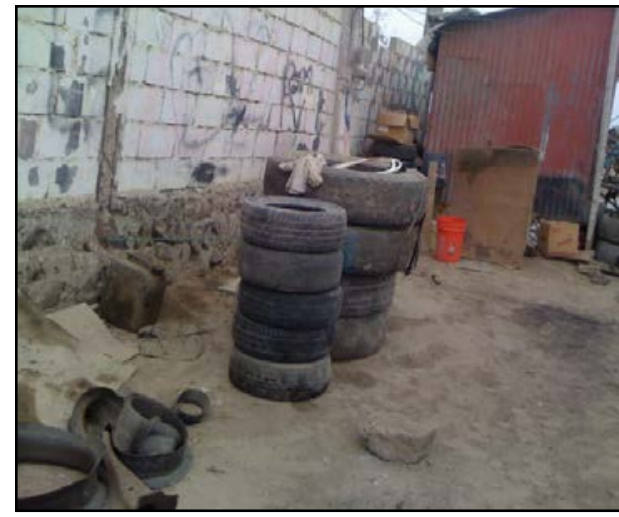

Foto 1.3.- Llantas acumuladas en vulcanizadoras vía La Libertad- Salinas.

Fuente Foto: Investigadores CIGEO-Julio/2014

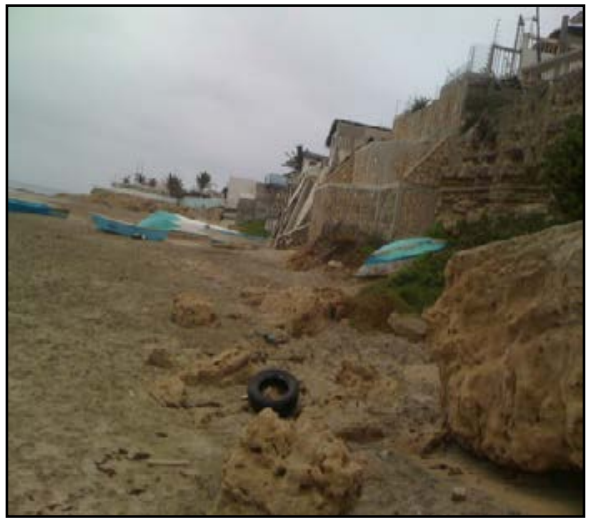

Foto 1.3.- Contaminación de llantas a la intemperie, Playa Ballenita.

Fuente Foto: Investigadores CIGEO-Julio/2014

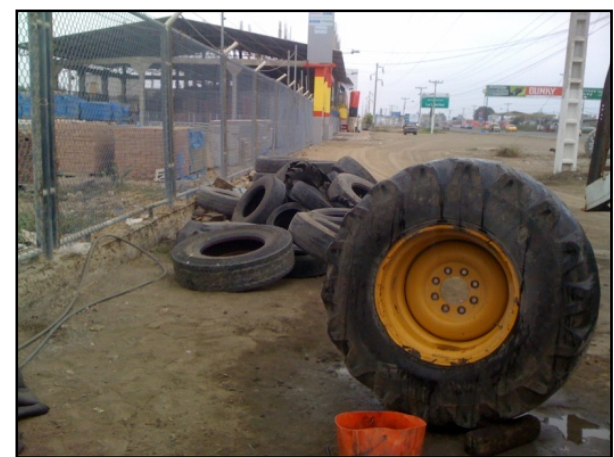

Foto 1.4.- Contaminación y acumulación de llantas a la intemperie, Vía La Libertad - Santa Elena.

Fuente Foto: Investigadores CIGEO-Julio/2014

La acumulación de neumáticos a la intemperie representa un importante inconveniente para la salud, como se puede observar en la foto 1.1, ya que en su interior se acumula agua lluvias, lo que se convierte en un 'foco' de reproducción de mosquitos causando enfermedades.

Otra repercusión del inadecuado procesamiento de estos desechos es que existen personas que quemarlos para evitar su acumulación sin considerar la polucíon del aire que producen. 
La creación de un diseño de hormigón con partículas provenientes de neumáticos, representa un material que, aunque puede ser hasta 40 por ciento más costoso, es altamente rentable, dado a que su duración puede ser cinco veces mayor. Este tipo de hormigón podría ser utilizado en: calles de bajo tránsito, banquetas y zonas peatonales, instalaciones deportivas, parques, pavimentos de estacionamientos tránsito ligero, sobre carpetas en estacionamientos, capas de nivelación en pisos o losas, área de albercas, estructuras sometidas a la abrasión hidráulica y mecánica.

\section{Metodología}

Para realizar el estudio de la elaboración de hormigones empleados en la construcción que minimicen el impacto al ambiente, empleando neumáticos usados de vehículos livianos y pesados como materia prima o agregados, se planteó la siguiente metodología dividida en 2 etapas:

Primera Etapa: Recopilación de información teórica y técnica, recopilación de muestras (selección de neumáticos usados y tipos de agregados) y encuestas a personas involucradas. En esta etapa se está realizando toda la recopilación de la información de los estudios originales y tabulación de datos obtenidos, ver gráfico 3.1.

a) Inspección visual y verificación in situ.

b) Levantamiento de información.

c) Revisión de información previa concerniente al tema de investigación.

d) Elaboración de modelos de hojas de cálculos para diseños preliminares posteriores.

e) Adquisición de materiales pétreos (arena, piedra) y cemento.

f) Ensayo de los agregados (arena, piedra y neumático triturado)

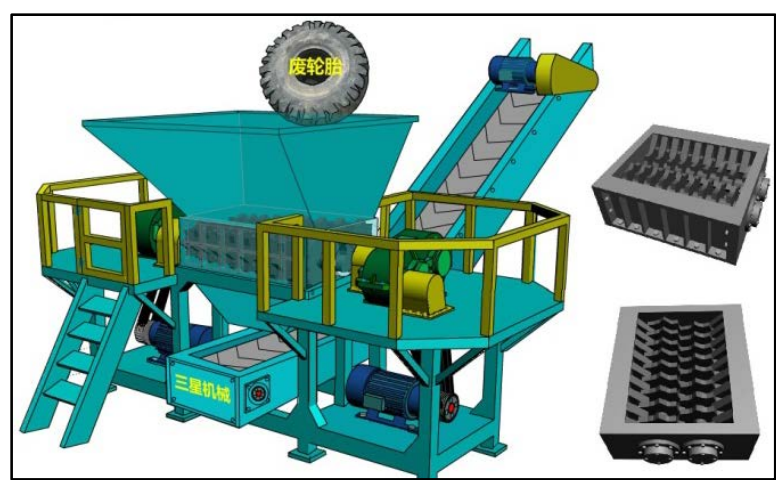

Gráfico 2.1.- Ejemplo de máquina trituradora cotizada. Fuente:http://us.sourcing.alibaba.com/rfq/request/rfq_view_quo tation_detail.htm?qid_vqd=253654487

Segunda Etapa: Diseño hormigón final en base a datos obtenidos en la primera etapa. a) Diseño de hormigón (con materiales pétreos) para la elaboración de probetas para ensayos.

b) Selección de porcentajes óptimos de cantidades de neumáticos triturados como agregados, para la elaboración de probetas según diseños.

c) Ensayos de resistencias de las probetas de hormigón.

d) Tabulación de resultados de resistencias de las probetas.

e) Evaluación y análisis de los resultados.

f) Propuesta y selección de diseño óptimo con agregado de neumático triturado.

\subsection{Metodología de trabajo y equipo utilizado}

\section{Levantamiento de Información}

Preliminarmente se realizó el levantamiento de información por medio de encuestas personales a los propietarios de las vulcanizadoras ubicadas en la Cabecera Cantonal de Santa Elena, en la parroquia Atahualpa, Chanduy y en la Ruta del Spondyllus del mismo cantón, siendo en total 16 los establecimientos encuestados.

El formato de la encuesta utilizada recogió datos estadísticos de la cantidad, tipos y destino final de los neumáticos que se atienden en los establecimientos intervenidos. Ver grafico 2.2.

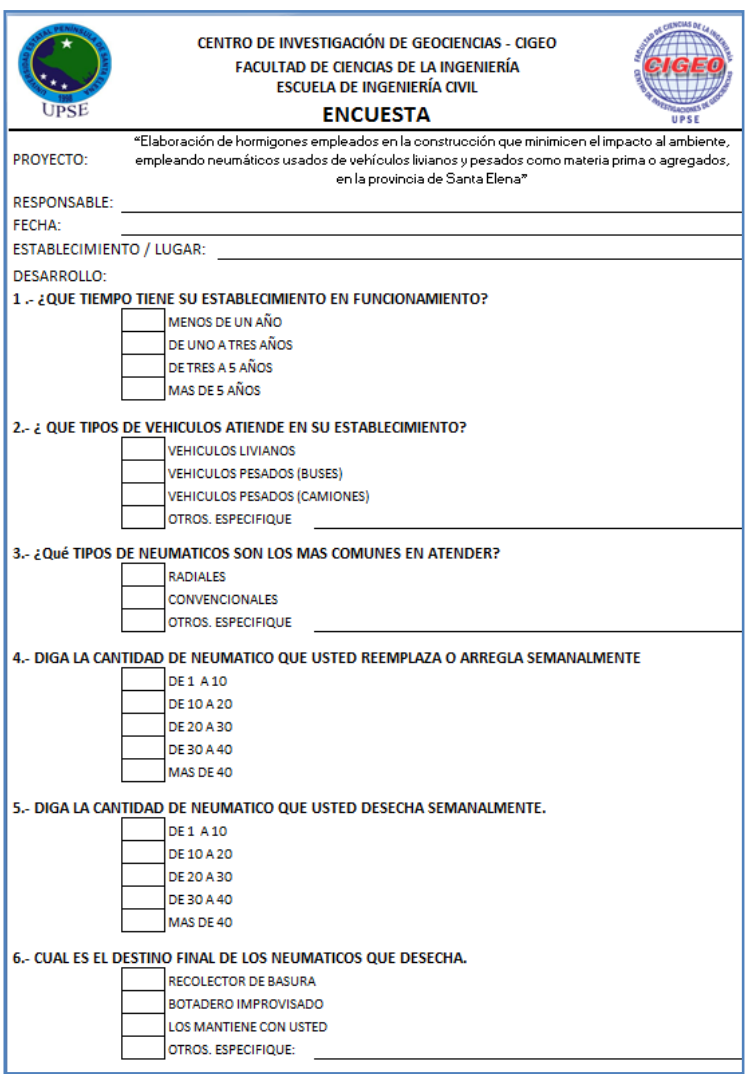

Gráfico 2.2.- Formato de Encuesta. Elaborado: Investigadores CIGEO. 
Posteriormente se harán este tipo de encuestas a vulcanizadoras de los cantones de La Libertad y Salinas. De esta manera se podrá recabar información general de la provincia y obtener datos más precisos y de interés local, que es uno de los puntos básicos esta investigación.

\section{Materiales de diseño}

Para un diseño de hormigón es necesario obtener una mezcla que posea determinadas propiedades tanto en estado fresco como endurecido, y que represente el menor costo de producción posible.

Según la Norma Técnica Ecuatoriana NTE INEN 1855, el hormigón es una mezcla de cemento Portland o cualquier otro cemento hidráulico, áridos (agregados), agua, con o sin aditivos, dosificado y mezclado según el tipo de estructura a edificar. Los agregados deben de tener un tamaño máximo limitado, además de características mecánicas, químicas y granulométricas. A esta mezcla puede añadírsele otros productos o materiales para mejorar algunas características determinadas (Adiciones y aditivos). A partir de este concepto se pueden añadir residuos triturados de neumáticos, que con una dosificación apropiada este dentro de los rangos permisibles de resistencia para un diseño de hormigón.

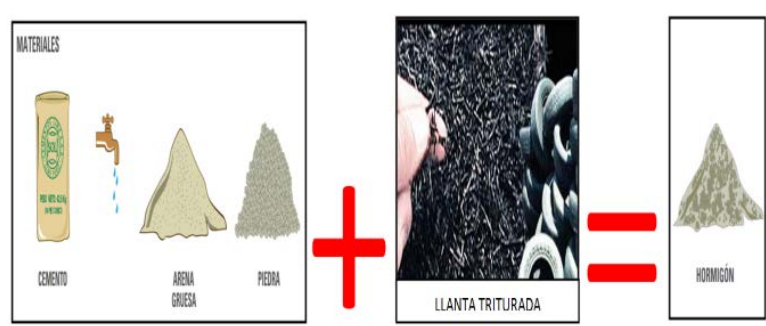

Gráfico 2.1.- Materiales de diseño de Hormigón. Fuente: http://www.progresol.com.pe ; http://www.100ideasparaemprender.com/home/2008/10/reciclaj e-de-llantas/

\section{Ensayos de los materiales}

Se define como ensayos de materiales a toda prueba física cuyo fin es determinar las propiedades mecánicas y químicas de un material, para verificar si cumple con los estándares establecidos de normas vigentes.

Se realizaran ensayos para el agregado grueso, agregado fino, residuos triturados de llantas usadas, los cuales se describirán posteriormente, y estos son:

- Granulometría.- consiste en tamizar el material (agregado) clasificándolo por tamaños con el fin de observar si se cumple especificaciones en las normas vigentes. El mismo que se hará al agregado grueso (piedra y neumáticos triturados) y fino (arena).
-Densidad, densidad relativa y absorción.- Estos ensayos determinaran la densidad, densidad relativa y porcentaje de absorción de los agregados a utilizar en el diseño de hormigón posterior.

-Peso especifico.- Este ensayo define el peso específico de un material en relación al peso del sólido del mismo y el peso del volumen de agua que dicho solido desaloja.

\subsection{Pre diseño de la mezcla}

Una vez realizado los ensayos a los materiales y teniendo los resultados de los mismos, se procede a seleccionar el método de diseño para la elaboración de probetas de hormigón. El método a emplear para la realización del pre diseño y diseño de hormigón con residuos triturados de neumáticos usados es el método propuesto por el ACI en la norma 211.1 - 70 (con ajustes de ACI-2008).

Para esto, se está elaborando hojas de cálculo mediante el utilitario EXCEL, para diseños de mezclas de hormigón.

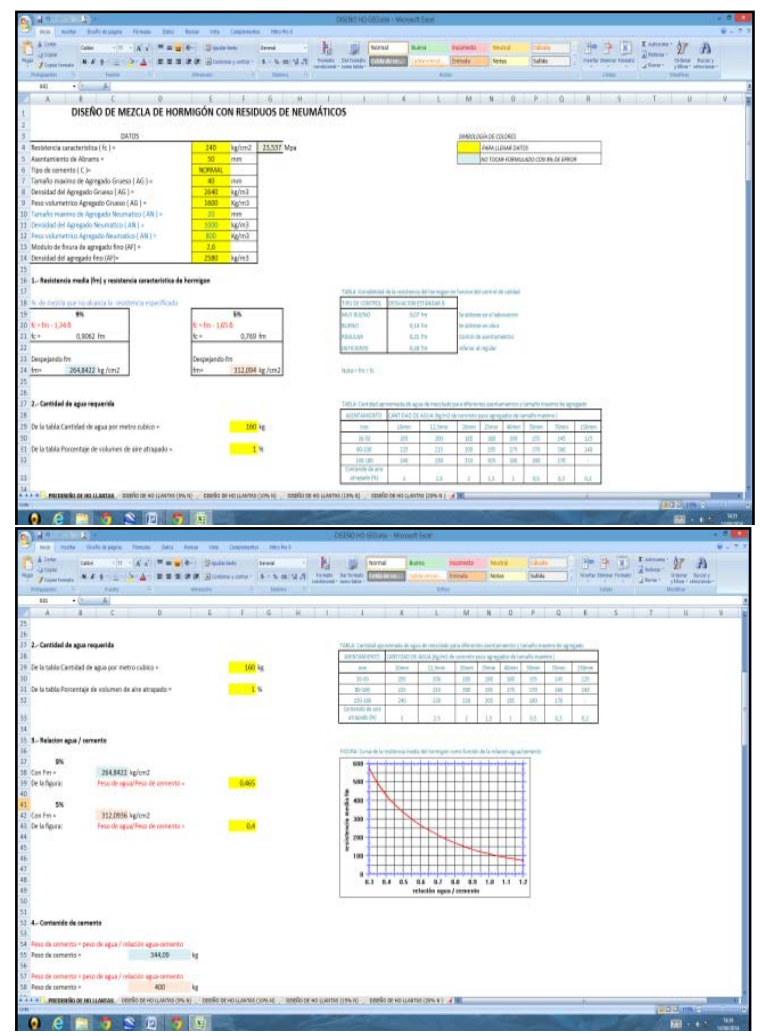

Gráfico 2.3.- Hoja de cálculo de pre-diseño de hormigón.

Fuente: Investigadores CIGEO

\section{Resultados preliminares.}

Las encuestas realizadas a las vulcanizadoras ubicadas en el cantón Santa Elena (Ver Tabla 1.1) una vez tabulas, mostraron los siguientes resultados: 
Tabla 1.1.- Vulcanizadoras encuestadas en el cantón Santa Elena.

Elaborado: Investigadores CIGEO.

\begin{tabular}{|c|c|c|}
\hline CANTón & LUGAR & $\begin{array}{c}\text { NOMBRE } \\
\text { ESTABLECEMENTO }\end{array}$ \\
\hline \multirow{19}{*}{ SANTA ELENA } & \multirow{6}{*}{$\begin{array}{l}\text { CABECERA } \\
\text { CANTONAL }\end{array}$} & V. SALINAS \\
\hline & & V. SAN JACDTO \\
\hline & & V. EL PAYASITO \\
\hline & & V. DARWIN \\
\hline & & V. VOLUNTAD DE DIOS \\
\hline & & V. SAN VICENTE \\
\hline & PARROQUIA & \multirow{2}{*}{ V. REYES } \\
\hline & ATAHUALPA & \\
\hline & \multirow{2}{*}{$\begin{array}{l}\text { PARROQUIA } \\
\text { CHANDUY }\end{array}$} & V. ERIKA \\
\hline & & LUBRICENTRO LINDAO \\
\hline & \multirow{2}{*}{$\begin{array}{l}\text { COMUNA } \\
\text { OLÓN }\end{array}$} & V. NIXON \\
\hline & & V. TIGRERO \\
\hline & COMUNA & \multirow[b]{2}{*}{ V. ORRALA } \\
\hline & $\begin{array}{l}\text { LIBERTADOR } \\
\text { BOLIVAR }\end{array}$ & \\
\hline & \multirow{2}{*}{$\begin{array}{l}\text { COMUNA } \\
\text { VALDIVIA }\end{array}$} & V. VIVIANA \\
\hline & & V. VALDIVIA \\
\hline & COMUNA & \multirow{2}{*}{ V. EL BRUJITO } \\
\hline & PALMAR & \\
\hline & $\begin{array}{l}\text { COMUNA SAN } \\
\text { PABLO }\end{array}$ & V. TALLER RAFA \\
\hline
\end{tabular}

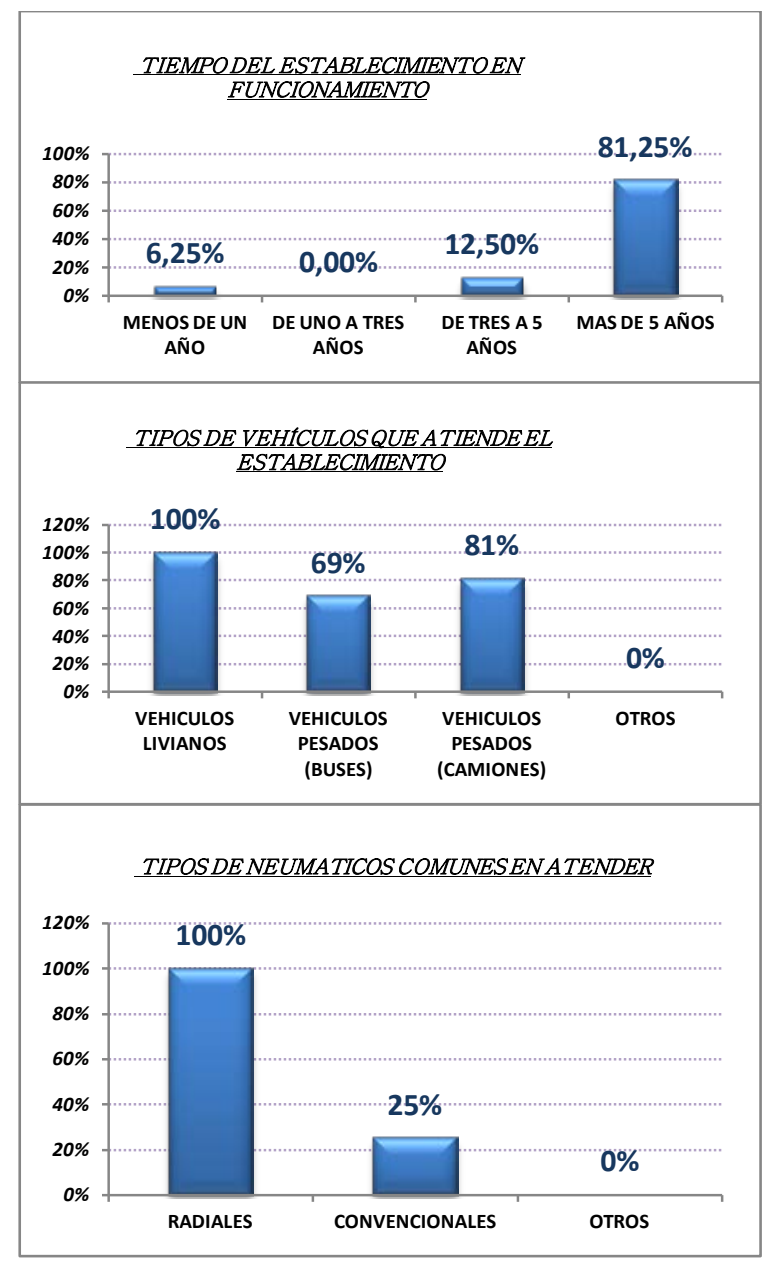

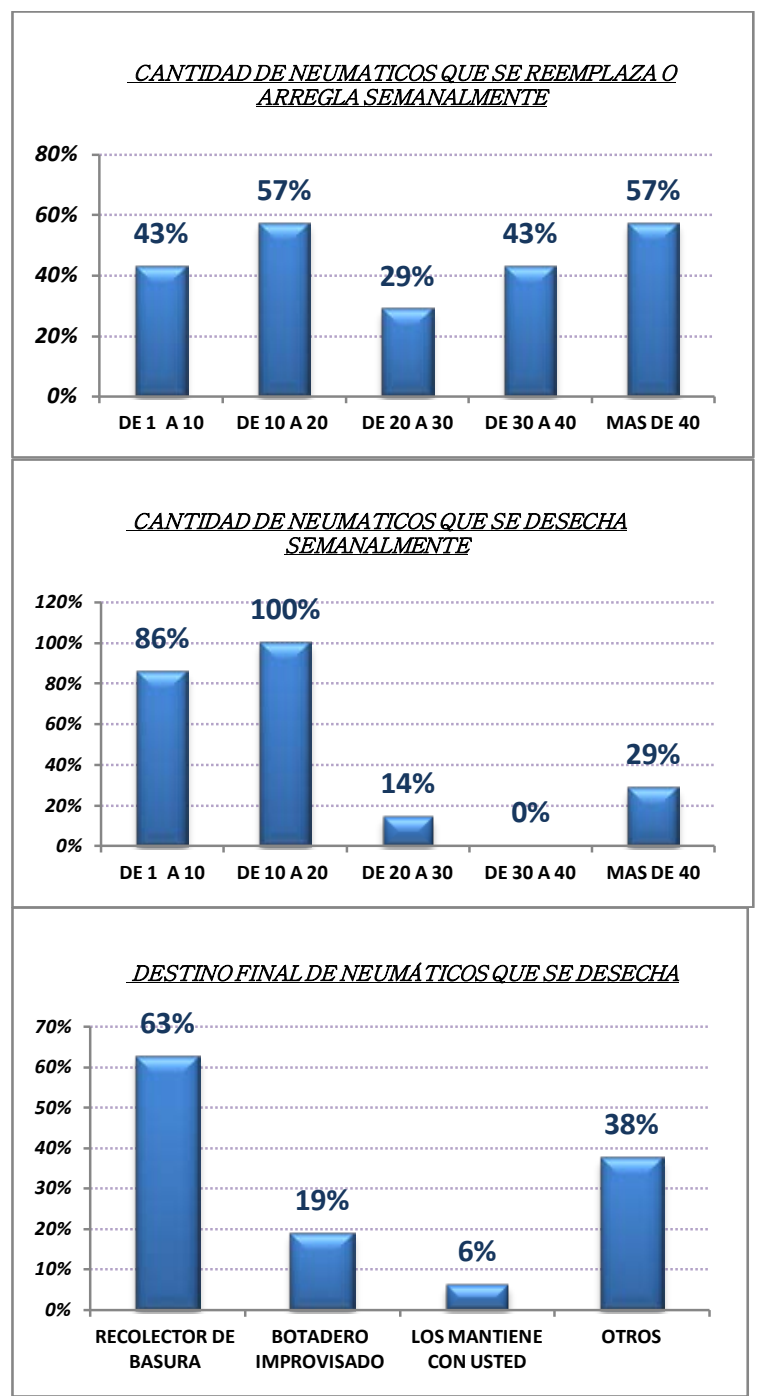

Gráfico 3.1.- Cuadros estadísticos de encuestas realizadas en el cantón Santa Elena. Elaborado: Investigadores CIGEO.

- La mayoría de establecimientos encuestados tiene en funcionamientos más de cinco años, los cuales atienden vehículos livianos y pesados, y el 75\% de estos atienden a su vez vehículos pesados (buses y camiones).

- El cien por ciento de los establecimientos atiende a vehículos con neumáticos radiales, y solo el 25\% atiende neumáticos del tipo convencionales.

- Las 19 vulcanizadoras encuestadas producen un aproximado de 240 llantas (desechos) semanalmente, proyectando al año un total de 11520 llantas; el 63\% de estos establecimientos los destina a los recolectores de basura, a su vez otro $38 \%$ los destina a compradores de reencauche, el $19 \%$ los envía a botaderos improvisados (terrenos baldíos) y el 6\% los mantiene en sus predios. 


\section{Conclusiones}

- En la actualidad existen en el mercado una mayor cantidad de neumáticos radiales en comparación a los convencionales.

- La mayor parte de los neumáticos que desechan las vulcanizadoras tienen como destino final el botadero municipal y el reencauche de las mismas.

- Considerando que solo en el cantón Santa Elena anualmente existe un desperdicio de 11520 neumáticos usados, este proyecto reduciría sustancialmente la contaminación que produce este tipo de desechos, además de proporcionar un uso adecuado en términos de construcción en la provincia de Santa Elena.

- Para obtener mejores resultados de la encuesta se debe tener información completa de los tres cantones de la provincia.

- En esta Primera Etapa se muestran los resultados iniciales de encuestas los cuales serán de utilidad para las actividades planteadas en la Segunda Etapa del proyecto.

\section{Referencias Bibliográficas}

Bibliografía:

[1]. Moreno, Víctor. Terreros, Carmen.(1995) Mecánica de Suelos. Quinta Edición. Guayaquil.

[2]. Romo, Marcelo. (2008). Hormigón armado. Quito, Ecuador.

[3]. ACI 318S-08,(2008). American Concrete Institute, Requisitos de reglamento para concreto estructural y comentarios.

[4]. Instituto ecuatoriano del cemento y del concreto, (2009). Notas Técnicas, Control de Calidad en el hormigón, Control por resistencia, Parte I. Primera Edición.

[5]. Polanco, Abraham (2010), Manual de Prácticas de Laboratorio de Concreto, Universidad Autónoma de ChihuahuaFacultad de Ingeniería.

[6]. Instituto Ecuatoriano de Normalización, (2010). Hormigón de Cemento Hidráulico. Determinación de la Resistencia a la compresión de especímenes cilíndricos de hormigón de cemento hidráulico. Primera Edición. Quito, Ecuador.

[7]. Carrasco, Ma. Fernada (2009).Santa Fe. Tecnologia del Hormigon-Ingenieria Civil.

Linkografía:

[8]. http://publiespe.espe.edu.ec/academicas/hor migon/hormigon02-a.htm

[9]. http://www.ingenierocivilinfo.com/2011/06/ modulo-de-elasticidad-del-hormigon.html

[10]. http://www6.uniovi.es/usr/fblanco/Tema8.M ateriales.Construccion.Hormigon.pdf

[11]. http://disensa.com/librogrande/libro/libro_gr ande.html

[12]. file://C:/Users/usuario/Downloads/Unidad_3 _ROCAS_Y_AGREGADOS_PARA_HORMI GONES.pdf

[13]. http://es.slideshare.net/yahil350/ensayo-demateriales-13447018\#

[14]. http://www.construmatica.com/construpedia/ Hormig\%C3\%B3n_Endurecido

[15]. http://civilgeeks.com/2011/12/11/propiedade s-principales-del-concreto/

[16]. http://ingevil.blogspot.com/2008/10/ensayocompresin-de-cilindros-de.html

[17]. http://www.ingenierocivilinfo.com/2011/06/ modulo-de-elasticidad-del-hormigon.html 